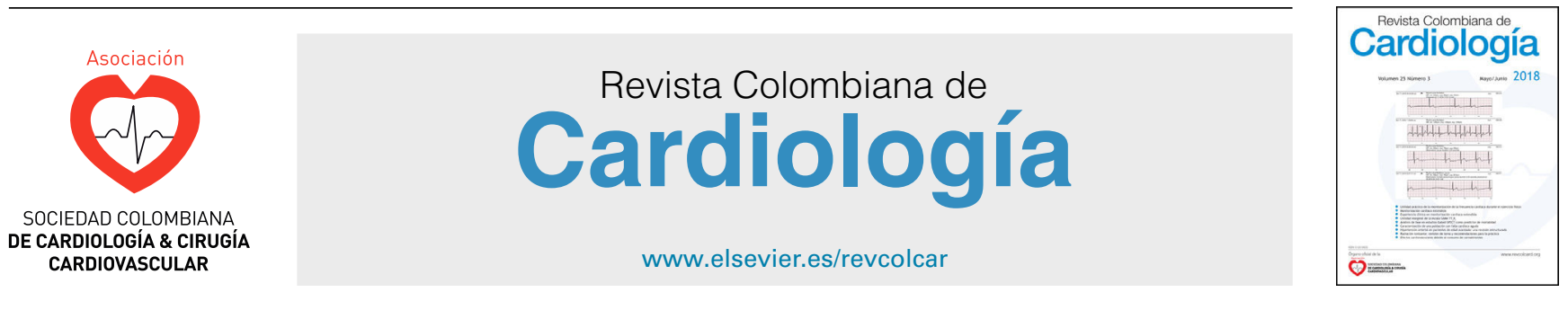

ARTÍCULO ESPECIAL

\title{
Tumores cerebrales y lesiones encefálicas por radiación ionizante
}

\author{
Víctor G. Aldana ${ }^{a, *}$, José F. Saaibi ${ }^{b}$ y Libardo A. Medina ${ }^{\mathrm{b}}$ \\ a Instituto Cardiovascular del Tolima, Clínica Nuestra, Ibagué, Colombia \\ ${ }^{b}$ Fundación Cardiovascular de Colombia, Bucaramanga, Colombia
}

Recibido el 12 de mayo de 2019; aceptado el 15 de septiembre de 2019

\section{PALABRAS CLAVE Exposición a radiación; Neoplasias cerebrales; Protección contra radiación}

\section{KEYWORDS}

Exposure to radiation; Brain tumours; Radiation protection

\begin{abstract}
Resumen En este documento se hace referencia a los tumores cerebrales por exposición a la radiación ocupacional y se abordan algunas técnicas de radioprotección usadas para el manejo de este riesgo. Reportes de caso han estimulado la preocupación entre operadores. El modelo anatómico de la localización de los tumores podría tener alguna asociación potencial con la exposición a la radiación. El uso de gorros quirúrgicos de radioprotección se ha incrementado entre intervencionistas; sin embargo, su efecto protector no es completo. El manejo apropiado del personal de salud involucrado y el riesgo al que se someten los pacientes debería estar siempre en la mente de los intervencionistas al momento de realizar procedimientos guiados por fluoroscopia.

(c) 2019 Publicado por Elsevier España, S.L.U. en nombre de Sociedad Colombiana de Cardiología y Cirugía Cardiovascular. Este es un artículo Open Access bajo la licencia CC BY-NC-ND (http:// creativecommons.org/licenses/by-nc-nd/4.0/).
\end{abstract}

\section{Brain tumours and encephalic lesions due to ionising radiation}

Abstract This article deals with brain tumours due to occupational radiation, as well as presenting some radiation protection techniques used to manage this risk. Case reports have increased concern among operators. The anatomical model for locating tumours could be associated with the exposure to the radiation. Although the use of radiation protective surgical caps has increased among interventionists, its protective effect is incomplete. The appropriate management of the health staff involved and the risk to which the patients are subjected should always be in the mind of the interventionists when they perform fluoroscopy-guided procedures.

(c) 2019 Published by Elsevier España, S.L.U. on behalf of Sociedad Colombiana de Cardiología y Cirugía Cardiovascular. This is an open access article under the CC BY-NC-ND license (http:// creativecommons.org/licenses/by-nc-nd/4.0/).

\footnotetext{
* Autor para correspondencia.

Correo electrónico: victorgaldana@gmail.com (V.G. Aldana).
} 
La exposición a la radiación ionizante ha venido siendo una de las principales preocupaciones en salud ocupacional entre especialistas que practican procedimientos guiados por fluoroscopia, especialmente, cardiólogos, radiólogos y profesionales aliados. Esta puede conllevar efectos estocásticos o determinísticos; estos últimos son más notables en lesiones en piel y desarrollo de cataratas, los cuales son causados por daño o muerte celular. La severidad del efecto se relaciona con la dosis una vez se ha logrado el umbral de dosis de radiación. Un efecto estocástico, o desarrollo de cáncer, se debe principalmente a un daño en la reparación del ADN, lo cual lleva a una transformación genética. La probabilidad de aparición aumenta con el total de la radiación absorbida por los tejidos; sin embargo, su severidad es independiente de la dosis total. Estos efectos generalmente son manifestados años o décadas después de la exposición ${ }^{1}$.

Comparado con otros órganos y tejidos, como colon, pulmones y médula ósea, el cerebro del adulto es considerado como uno de los tejidos menos radiosensibles, debido a la condición no mitotótica de sus células. Típicamente la cabeza no está protegida durante los procedimientos intervencionistas guiados por fluoroscopia, en parte debido a la baja radiosensibilidad del cerebro.

Estimaciones recientes indican que el cerebro está expuesto entre diez y veinte veces más a la radiación del cuerpo que está protegido por el uso de los chalecos plomados. La dosis anual que recibe el cerebro se encuentra entre 20 y 30 mSv, especialmente mayor cuando no se usan medidas de protección adicionales como la mampara plomada. Por tanto, se estima una media de exposición a la radiación ionizante durante un trabajo promedio en intervencionismo moderado de 25 años de 1 a $3 \mathrm{~Sv}^{2,3}$.

No obstante, un número reciente de estudios han reportado tumores cerebrales en profesionales de la salud quienes están crónicamente expuestos a la radiación ionizante (cardiólogos intervencionistas y radiólogos intervencionistas $\mathrm{cl} / \mathrm{RI}$ ), con lo que se aumenta hasta en dos veces el riesgo de muerte por cáncer cerebral comparado con controles no expuestos $^{4-6}$. Estos hallazgos han elevado la preocupación en cuanto al riesgo para la salud de cardiólogos intervencionistas quienes están expuestos, sin precedentes, a niveles altos de radiación ionizante ocupacional ${ }^{7}$.

La exposición a la radiación ionizante es un riesgo demostrado para pacientes y de potencial peligro para médicos intervencionistas $(\mathrm{Cl} / \mathrm{RI})$ durante procedimientos invasivos guiados por fluoroscopia en salas de hemodinamia. Se han descrito efectos potenciales determinísticos y estocásticos a la exposición a altas dosis de radiación ionizante; sin embargo, los efectos a largo plazo de exposición a bajas dosis son menos conocidos ${ }^{8}$.

Estudios previos estiman que en este escenario puede haber riesgo mayor de malignidad para el personal de las salas de cateterismo cardiaco ${ }^{9,10}$. En la última década se han reportado casos cada vez más frecuentemente de tumores de cabeza y cuello afectando a médicos intervencionistas $(\mathrm{Cl} / \mathrm{RI})$. La mayoría de los afectados fueron diagnosticados con glioblastoma multiforme maligno (55\%), dos casos de astrocitoma y cinco casos de meningioma; predominó el compromiso del lado izquierdo del cerebro. Todas estas neoplasias malignas han sido asociadas con exposición a la radiación ${ }^{11,12}$.
Roguin et al. reportaron 31 tumores de cabeza y cuello en intervencionistas cardiólogos y radiólogos y dos otras especialidades expuestas, de los cuales 22 fueron del lado izquierdo (85\%), que usualmente es el lado más expuesto de la cabeza del operador durante el procedimiento. Los autores sugieren que esta incidencia en intervencionistas avala una relación con la exposición ocupacional en esta cohorte. Pese a ello, hasta el momento no se ha determinado con certeza una relación directa entre la exposición a radiación del operador y la aparición de cáncer cerebral, pero los reportes recientes han aumentado la expectativa ante una posible asociación ${ }^{4}$.

La exposición del operador a radiación es dependiente de la radioprotección usada (chalecos plomados), la posición, la distancia desde la fuente de radiación y los factores inherentes al paciente. Los avances tecnológicos en equipos de radioprotección y el uso de estos han reducido la exposición a la radiación dispersa, pero en cuanto a la utilización de gorros plomados o de protección cerebral esta ha sido limitada, probablemente debido a desconocimiento de su beneficio y pobre tolerancia a su aplicación ${ }^{13}$.

En el estudio BRAIN, recientemente publicado por Reeves et al. se demostró que cardiólogos intervencionistas, tanto experimentados como en entrenamiento, están sustancialmente expuestos a la radiación ionizante al lado izquierdo del cerebro, en comparación con el lado derecho, considerando incluso que el estudio se llevó a cabo en centros universitarios con equipos de angiografía de última generación y, de la misma manera, con medidas de protección, como gorros y mamparas plomadas, los cuales pueden disminuir el porcentaje de exposición a niveles similares a las del medio ambiente ${ }^{14}$.

Reportes recientes de cáncer del lado izquierdo del cerebro en operadores de procedimientos guiados por fluoroscopia son alarmantes dada la naturaleza agresiva y la localización de estos tumores; además, los intervencionistas son algunos de los miembros más expuestos a radiación entre el gremio médico ${ }^{15}$.

Según los resultados obtenidos de investigaciones previamente publicadas se estima que la media de tiempo de práctica en Cardiología intervencionista antes del diagnóstico de neoplasias cerebrales es de aproximadamente $23+5$ años ${ }^{5}$.

Borguini et al. basaron su estudio acerca del papel de las moléculas de ARN no codificante (microARN miARN), las cuales se han encontrado previamente desreguladas en muchas enfermedades. La estabilidad y especificidad a tejidos de miARN las hacen un biomarcador ideal para determinar posibles asociaciones entre la exposición a bajas dosis de radiación y el potencial riesgo de enfermedades ${ }^{16}$.

El miR-134 ha sido identificado como específico del cerebro, está implicado en el desarrollo de la sinapsis y es fundamental en el aprendizaje y la memoria. Su desregulación se ha encontrado también en demencias (enfermedad de Alzheimer), epilepsia del lóbulo temporal, trastorno afectivo bipolar y tumores cerebrales, como oligodendrogliomas y glioblastomas. En adición, el miR-134 está asociado con varios genes mediadores en la proliferación de células cancerígenas; se ha demostrado una regulación a la baja en tumores del glioma y, de la misma manera, una sobreexpresión que inhibe el crecimiento celular del glioma a través del KRAS activando el camino del ERK. 
Igualmente, se ha confirmado que su expresión estuvo disminuida significativamente en glioblastoma ${ }^{17,18}$.

En este mismo estudio, luego de comparar un grupo de cardiólogos intervencionistas expuestos a radiación con un grupo control, en 41 intervencionistas se logró realizar la reconstrucción de su dosimetría con una media de exposición de 16 años y una media de dosis efectiva individual de $19 \mathrm{mSv}$ (RI 11-43 mSv). En el microanálisis se encontraron 4 microARN alterados (miR-134, miR-575, miR-127 y miR-2392), de los cuales el miR-134 y el miR2392, específicos del cerebro, estaban significativamente desregulados. Luego de un modelo de regresión múltiple solo la exposición a radiación ionizante fue el predictor significativo de desregulación de ambos microARN. Posteriormente, los cardiólogos intervencionistas fueron divididos entre exposición baja $(n=20)$ y alta $(n=21)$ sobre la dosis media de exposición efectiva acumulada ( $\geq 19 \mathrm{mSv}$ ); el miR-134 específico del cerebro se encontró significativamente desregulado en cardiólogos intervencionistas altamente expuestos ( $p 0,004)$. Hasta el momento se desconoce la función del miR-2392; sin embargo, estudios recientes han mostrado su desregulación en cáncer gástrico.

Por consiguiente, la desregulación significativa del miR134 específico del cerebro sugiere que el daño cerebral es uno de los principales potenciales riesgos a largo plazo de la exposición a radiación ionizante sin protección cerebral en cardiólogos intervencionistas, así como también puede generar consecuencias en cuanto a deterioro cognitivo ${ }^{19}$, las cuales han sido reportadas por Maraziti et al. En un test neurosicológico realizado con médicos expuestos y no expuestos se observó deterioro en la memoria verbal a largo plazo en el grupo expuesto en una habilidad que es principalmente modulada por el hemisferio izquierdo del hipocampo ${ }^{20}$.

Pese a lo anterior, hasta el momento no existe evidencia contundente que demuestre que la exposición a largo plazo a la radiación ionizante de uso médico incremente el riesgo de cáncer cerebral; de igual forma, en la actualidad no es posible establecer una asociación directa entre la exposición del operador y el riesgo de neoplasias cerebrales. Por ende, se requieren, con urgencia, nuevos estudios para investigar este potencial riesgo exposicional entre intervencionistas y mantener la recomendación del uso sistemático de medidas de protección a la radiación ionizante con efecto demostrado.

\section{Conflicto de intereses}

Ninguno.

\section{Bibliografía}

1. Miller DL, Balter S, Schueler BA, Wagner LK, Strauss KJ, Vaño E. Clinical radiation management for fluoroscopically guided interventional procedures. Radiology. 2010;257:321-32.

2. International Commission on Radiological Protection. The 2007 Recommendations of the International Commission on Radiological Protection. ICRP publication 103. Ann ICRP. 2007;37:1-332.
3. Marsh RM. Fluoroscopy operators' brains and radiation. JACC Cardiovasc Interv. 2016;9:301.

4. Roguin A, Goldstein J, Bar O. Brain tumours among interventional cardiologists: a cause for alarm? Report of four new cases from two cities and a review of the literature. Eurolntervention. 2012;7:1081-6.

5. Roguin A, Goldstein J, Bar O, Goldstein JA. Brain and neck tumors among physicians performing interventional procedures. Am J Cardiol. 2013;111:1368-72.

6. Rajaraman P, Doody MM, Yu CL, Preston DL, Miller JS, Sigurdson AJ, et al. Cancer Risks in U.S Radiologic technologists working with fluoroscopically guided interventional procedures, 19942008. Am J Roentgenol. 2016;206:1101-8.

7. Klein LW, Bazavan M. The economic imperatives underlying the occupational health hazards of the cardiac catheterization laboratory. Circ Cardiovasc Interv. 2016;9: e003742.

8. Klein LW, Miller DL, Balter S, Laskey W, Haines D, Norbash A, et al. Occupational health hazards in the interventional laboratory: time for a safer environment. Catheter Cardiovasc Interv. 2009;73:432-8.

9. Venneri L, Rossi F, Botto N, Andreassi MG, Salcone N, Emad A, et al. Cancer Risk from professional exposure in staff working in cardiac catheterization laboratory: Insights from the National Research Councilś Biological Effects of lonizing Radiation VII Report. Am Heart J. 2009;157:118-24.

10. Picano $E$, Vano $E$. The radiation issue in cardiology: the time for action is now (abstr.). Cardiovasc Ultrasound. 2011;9:35.

11. Bondy ML, Scheurer ME, Malmer B, Barnholtz-Sloan JS, Davis FG, llýasova $D$, et al. Brain tumor epidemiology: consensus from the Brain Tumor Epidemiology Consortium. Cancer. 2008; 113:1953-68.

12. Sadetzki S, Flint-Richter P, Ben-Tal T, Nass D. Radiationinduced meningioma: a descriptive study of 253 cases. J Neurosurg. 2002;97:1078-82.

13. Roguin A. CardioPulse. Radiation in cardiology: can't live without it!: using appropriate shielding, keeping a distance as safely as possible and reducing radiation time are essential principles for radiation reduction. Eur Heart J. 2014;35: 599-600.

14. Reeves R, Ang L, Bahadorani J, Naghi J, Dominguez A, Palakodeti $\mathrm{V}$, et al. Invasive cardiologists are exposed to greater left sided cranial radiation The BRAIN Study. JACC Cardiol Int. 2015;8:1197-206.

15. Delichas M, Psarrakos K, Molyvda- Athanassopoulou E, Giannoglou G, Sioundas A, Hatziioannou K, et al. Radiation exposure to cardiologists performing interventional cardiology procedures. Eur J Radiol. 2003;48:268-73.

16. Esteller M. Non-coding RNAs in human disease. Nat Rev Genet. 2011;12:861-74.

17. Pan JY, Zhang F, Sun CC, Li SJ, Li G, Gong FY, et al. miR134: a human cancer suppressor? Mol Ther Nucleic Acids. 2017;6:140-9.

18. Schratt GM, Tuebing F, Nigh EA, Kane CG, Sabatini ME, Kiebler $\mathrm{M}$, et al. A brain-specific microRNA regulates dendritic spine development. Nature. 2006;439:283-9.

19. Borghini A, Vecoli C, Mercuri A, Carpeggiani C, Piccaluga E, Guagliumi G, et al. Low-dose exposure to ionizing radiation deregulates the brain-specific microRNA-134 in Interventional Cardiologists. Circulation. 2017;136:2516-8.

20. Marazziti D, Tomaiuolo F, Dell'Osso L, Demi V, Campana S, Piccaluga $\mathrm{E}$, et al. Neuropsychological testing in interventional cardiology staff after long-term exposure to ionizing radiation. J Int Neuropsychol Soc. 2015;21:670-6. 